ISSN 1991-8631

Original Paper

http://indexmedicus.afro.who.int

\title{
Structure et composition floristiques des agroforêts à base de caféiers arabica (Coffea arabica L.) dans les hauts plateaux de l'Ouest du Cameroun
}

\author{
Mbarga Amougou MANGA ${ }^{1 *}$, Amougou AKOA ${ }^{2}, \mathrm{D}_{\text {. ABOLO }}{ }^{3}$, Amang A MBANG $^{3}$, \\ $\mathrm{JA} \mathrm{Mouen} \mathrm{BEDIMO}^{4}$, LE Bidzanga NOMO ${ }^{5}$ et Neba Derick AKUME ${ }^{6}$ \\ ${ }^{I}$ IRAD, Station de Foumbot, Foumbot, Cameroun. \\ ${ }^{2}$ Université de Yaoundé I, Faculté des Sciences, Département de Biologie et \\ Physiologie Végétales, Yaoundé, Cameroun. \\ ${ }^{3}$ IRAD, Centre de Nkolbisson, Yaoundé, Cameroun. \\ ${ }^{4}$ IRAD, Direction Générale, Yaoundé, Cameroun. \\ ${ }^{5}$ IRAD, Direction de la Recherche Scientifique, Yaoundé, Cameroun. \\ ${ }^{6}$ IRAD, Antenne de Santa, Bamenda, Cameroun. \\ *Auteur correspondant ; E-mail : akoumoumanga@yahoo.fr; Tel.: (+237) 76190206
}

\section{RESUME}

Un état floristique des agroforêts à base de caféiers arabica a été effectué dans les Hauts plateaux de l'Ouest du Cameroun. Il a porté sur 100 parcelles réparties dans quatre niveaux d'altitudes : basse $(<1200 \mathrm{~m})$, moyenne inférieure (1200-1450 m), moyenne (1450-1700 m) et haute altitudes ( $\geq 1700 \mathrm{~m})$. L'analyse de la variance au test de Fisher a révèlé une influence significative de l'altitude sur la densité des groupes végétaux. En moyenne (2314 plants/ha) et haute altitudes (2181 plants/ha), les caféiers arabica y sont plus denses; les Musacées sont plus denses en altitude moyenne inférieure (695 plants/ha). Persea americana est la plus dense en altitude intermédiaire; mais en basse et haute altitudes, se trouvent respectivement Leucaena leucocephala (72 plants/ha) et Raphia mabilensis (27 plants/ha). La structure diamétrique des espèces ligneuses prend l'allure d'une cloche, au desssus de $1200 \mathrm{~m}$. Dacryodes edulis, $P$. americana, Cola spp et $R$. mabilensis sont les seules présentant une occurrence $\geq 10.14 \%$ des agroforêts renferment au moins un plant de Cola spp., D. edulis, P. americana et une touffe de R. mabilensis. En dessous de 1450 m, D. edulis accroît écologiquement son importance; par contre au niveau supérieur, Cola. spp. et $P$. americana sont les plus importantes.

(C) 2013 International Formulae Group. All rights reserved.

Mots clés : Agroforêts, Coffea arabica, altitude, densité, occurrence, importance.

\section{INTRODUCTION}

De par sa diversité géoclimatique, le Cameroun cultive le café robusta (Coffea canephora $\mathrm{P}$.) en altitude $\leq 1000 \mathrm{~m}$ et le café arabica (C. arabica L.) en altitude $>1000 \mathrm{~m}$. La volatilité des cours mondiaux de café et la récession économique du secteur agricole ont contribué à dégrader l'économie nationale au fil des ans. La production caféière continue de suivre une régression : celle du café arabica en occurrence était de 2499 t en 2011, soit 7 - 11 fois moins que dans les années 1980 (Anonyme, 2012).

La très forte pression foncière $(168$ $\mathrm{hbts} / \mathrm{km}^{2}$ ) observée dans les Hauts plateaux de l'Ouest du Cameroun (HPO) a réduit les 
superficies caféières (Fotsing, 1999a, 1999b) et Palmales (Kaffo et al., 2007). De plus, en l'absence de recommandations techniques adaptées à leurs circonstances, la majorité des caféiculteurs limite le risque de ne plus subvenir aux divers besoins de leurs ménages, soit en accélérant une tendance ancienne à la diversification agricole, soit en procédant à un abandon progressif de la caféiculture substituée à de nouveaux modes de production culturales et/ou arboricoles (Kaffo et al., 2007 ; Uwizeyimana, 2009); et dont les contraintes correspondent mieux à leurs possibilités techniques et financières (Guétat-Bernard, 2011).

En dépit de cette situation, la nouvelle politique agricole nationale en rapport avec la production caféière est axée sur la relance de cette filière (Anonyme, 2009). Dans le cadre de la rénovation et l'extension des vergers, la disponibilité en technologies appropriées nécessite l'acquisition des informations relatives à la structure et à la composition floristiques.

En Amérique du Sud, les connaissances en termes d'état des lieux floristiques des caféières arabica sont éloquentes (Soto-Pinto et al., 2000, 2001; Vaast et Harmand, 2002; Hall, 2003 ; Boulay, 2004); mais au Cameroun, elles sont limitées. Une étude du MINAGRI (Anonyme, 1981) souligne que, $77,5-83 \%, 2,9-5 \%$ et $0,7-$ $4,2 \%$ des caféières arabica sont respectivement sous Musa spp., fruitiers et aménagées. Bouharmont (1981 ; 1992) préconise une densité caféière de 2222 plants/ha en basse altitude (1000 - $1450 \mathrm{~m}$ ) et de 2500-2666 plants/ha en altitude supérieure $(\geq 1450 \mathrm{~m})$ dans les HPO du Cameroun. La présente étude vise donc à effectuer un état des lieux floristiques des agroforêts à base de caféiers arabica dans les HPO.

\section{MATERIELS ET METHODES}

Zone d'étude : Hauts plateaux de l'Ouest du Cameroun

La zone d'étude couvre les régions de l'Ouest (13 $872 \mathrm{~km}^{2}$ ) et une partie de celle du Nord-Ouest du Cameroun. Ces dernières s'identifient, d'une part, par une démographie galopante, se situant largement au premier rang des régions africaines à forte pression démographique $168 \mathrm{hbts} / \mathrm{km}^{2}$. Et d'autre part, par une topographie contrastée et articulée (Fotsing, 1999b) :

- au Sud, le plateau granito-gneissique (1200$1400 \mathrm{~m}$ d'altitude) avec des reliefs poly convexes;

- au Sud-Ouest, des montagnes modestes de 2100 m d'altitude ;

- au Nord, le plateau basaltique (1400-1600 m d'altitude) avec topographie plus calme ;

- vers le Nord-Ouest, le massif volcanique de Bamboutos (2700 m d'altitude) ;

- et, la plaine du Noun longeant la bordure orientale du plateau bamoun.

En contrebas de cet ensemble, ressort un gigantesque escarpement donnant lieu à la plaine de Mbos. Quatre principaux cours d'eau y arpentent (Mbam, Ndé, Nkam et Noun). Le climat est de type subéquatorial de mousson à dominance humide et fraîche, fortement influencé par l'orientation des pentes et l'altitude (> $1500 \mathrm{~mm} / \mathrm{an}$ ).

$\mathrm{La}$ végétation est profondément marquée par l'altitude : les savanes arborées et arbustives à Annona senegalensis et Terminalia glaucescens, et les savanes herbeuses à Afromomum latifolium et Imperata cylindrica ; les vestiges de la forêt littorale ; les galeries forestières et les formations montagnardes (Podocarpus latifolius, P. milanjianus ...). Les sols (ferralitiques, peu évolués et hydromorphes) sont remarquables par leur fertilité originelle, mais vulnérables après culture.

\section{Choix et caractérisation des caféières}

Sur la base des études de Fotsing (1999b) portant sur l'érosion et la conservation des sols des HPO et suivant les recommandations actuelles de la vulgarisation agricole, la zone d'étude a été stratifiée en quatre niveaux d'altitude : la basse altitude (<1200 m) ou Altitude I, l'altitude moyenne inférieure (1200-1450 m) ou Altitude II, la moyenne 
altitude (1450-1700 m) ou Altitude III et la haute altitude $(\geq 1700 \mathrm{~m}$ ) ou Altitude IV. Les zones d'altitude inférieure et supérieure regroupent respectivement les zones d'altitude I-II et III-IV.

chaque niveau d'altitude, nous avons opéré un choix des villages où la caféiculture arabica est prioritaire; ainsi lors des entretiens avec les groupes de caféiculteurs sur le choix des caféières, 25 caféières régulièrement entretenues ont été aléatoirement sélectionnées par niveau d'altitude, tout en tenant compte de l'accès au site.

La caractérisation a consisté en une prise des coordonnées géographiques des parcelles à l'aide d'un GPS. Par la suite, des transects de $40 \mathrm{x}$ $5 \mathrm{~m}$ (Gillison et al., 2004; Sonwa, 2004) ont été aménagés en double diagonale (soit $400 \mathrm{~m}^{2}$ ). Sur les relevés floristiques, sont systématiquement recensées les espèces ligneuses et agricoles pérennes (en termes de présence, diamètre au collet ou 1,30 m du sol, hauteur...). Compte tenu des diverses strates présentes dans les agroforêts à base de caféiers arabica et de leurs diverses importances, les espèces en association ont été regroupées en cinq : caféiers, Musacées, fruitiers, autres arbres et Palmales.

\section{Traitements et analyses des données}

Les données collectées ont été saisies, regroupées et classées afin de :

- déterminer la distribution de la densité moyenne, de la structure diamétrique et de la surface terrière des divers groupes végétaux ;

- déterminer la distribution de l'occurrence parcellaire, la dispersion et la concentration des espèces végétales associées;

- déterminer l'abondance et l'importance relative des espèces et des familles botaniques associées.

L'estimation de certaines variables a porté sur une analyse descriptive et sur des analyses statistiques avec GLM-SAS au seuil de signification de 5\%. Pour un test significatif, une comparaison des moyennes est établie avec Student-Newman Keuls.
Méthodes d'analyses

\section{Caractéristiques structurales: Densité moyenne, structure diamétrique et surface terrière}

La densité moyenne des plants (D) représente : Nombre de plants / Surface totale échantillonnée (plants/ha).

La structure diamétrique des caféiers et des espèces ligneuses recensées a été établie sur la base d'un regroupement par classe $(\mathrm{cm})$. Les caféiers sont représentés dans 6 classes de diamètre $(<5 \mathrm{~cm}, 5-6 \mathrm{~cm}, 6-7 \mathrm{~cm}, 7-8 \mathrm{~cm}, 8-9$ $\mathrm{cm}, \geq 9 \mathrm{~cm})$; par contre, les autres espèces ligneuses le sont dans 10 classes : $<10 \mathrm{~cm}, 10-$ $20 \mathrm{~cm}, 20-30 \mathrm{~cm}, 30-40 \mathrm{~cm}, 40-50 \mathrm{~cm}, 50-60$ $\mathrm{cm}, 60-70 \mathrm{~cm}, 70-80 \mathrm{~cm}, 80-90 \mathrm{~cm}$ et $\geq 90 \mathrm{~cm}$.

La surface terrière $(\mathrm{G})$ est calculée ainsi (Latreille et al., 2003):

$$
\mathrm{G}=\Pi \mathrm{D}^{2} / 4\left(\mathrm{~m}^{2} / \mathrm{ha}\right) ;
$$

$\mathrm{D}$ : diamètre au collet ou à hauteur de poitrine.

\section{Composition floristique: Occurrence parcellaire, dispersion et concentration}

L'occurrence parcellaire représente le nombre de parcelles apparaissant pour une espèce donnée dans une zone (Latreille et al., 2003) ;

Fréquence spécifique $(\%)=$ Nombre total d'espèces apparues par occurrence / Nombre total d'espèces observées ;

$\mathrm{La}$ dispersion (contraire de la concentration) représente le nombre d'apparitions des individus d'une espèce donnée couvrant une importante occurrence parcellaire dans une zone.

\section{Importance relative des espèces (IVI)}

Cet indice écologique combine trois indicateurs d'une espèce x (Guedje et al., 2002 ; Latreille et al., 2003) :

Densité relative $(\mathbf{D R x})=$ Nombre d'individus de l'espèce $\mathrm{x} /$ Nombre total d'individus observés (\%) 
Dominance relative $($ DORx $)=\Sigma$ Surface terrière de l'espèce $\mathrm{x} /$ Surface terrière totale $(\%)$

Fréquence relative $(\mathbf{F R x})=$ Nombre d'occurrences de l'espèce $\mathrm{x} /$ Nombre total d'occurrences (\%)

$\mathbf{I V I}=\mathrm{DRx}+\mathrm{DORx}+\mathrm{FRx}$

\section{Importance relative des familles (FIV)}

Il représente plutôt un IVI calculé pour toutes les espèces appartenant à une même famille botanique A (Guedje et al., 2002 ; Latreille et al., 2003)

Diversité relative $($ DIRA $)=$ Nombre d'espèces de la famille A / Nombre total d'espèces observées (\%)

Dominance relative $($ DORA $)=$ Surface terrière de la famille A / Surface terrière totale (\%)

Densité relative $($ DRA $)=$ Nombre total d'individus de la famille A / Nombre total d'individus observés (\%)

$\mathbf{F I V}=$ DIRA + DORA + DRA

\section{RESULTATS}

\section{Densité moyenne}

L'analyse de variance au test de Fisher (Figure 1) révèle l'influence significative de l'altitude sur la variation de la densité moyenne de caféiers (Pr < 0,0001). La comparaison des moyennes montre qu'il n'y a pas une différence significative entre les niveaux de moyenne (2314 plants/ha) et haute altitudes (2181 plants/ha), lesquelles se classent parmi les densités les plus élevées.

Comme pour les caféiers, l'altitude influence significativement la densité des autres groupes végétaux $(\operatorname{Pr}<0,0001)$. Les Musacées sont plus denses en altitude moyenne inférieure (695 plants/ha); par contre, les fruitiers se distinguent par des densités significativement élevées et statistiquement identiques (77 et 92 plants/ha) en altitude intermédiaire.
Quant aux autres arbres, leur densité est significativement plus dense en basse altitude (120 plants/ha); par contre en altitude supérieure, leur densité est statistiquement identique et faible (32 et 25 plants/ha).

La densité des Palmales dans les caféières de haute altitude (27 touffes/ha) est significativement supérieure à celle de basse altitude (7 touffes/ha) qui est statistiquement identique à celle des caféières d'altitude intermédiaire (Figure 1).

Globalement, les agroforêts à base de caféiers arabica sont plus denses en basse altitude (186 plants/ha) comparativement aux autres niveaux. Parmi les espèces associées, Persea americana est la plus dense en altitude intermédiaire ( 24 et 30 plants/ha) ; mais en basse et haute altitudes, se présentent respectivement Leucaena leucocephala (93 plants /ha) et Raphia mabilensis (27 plants /ha).

En association, les Mimosaceae (103 plants /ha) et les Burseraceae (18 plants /ha) sont les familles les plus denses en basse altitude ; en altitude moyenne inférieure, ce sont les Lauraceae (23 plants/ha) et les Burseraceae (24 plants/ha). En altitude moyenne, ces dernières se retrouvent associées aux Sterculiaceae (25 plants /ha); mais en haute altitude, les Arecaceae (27 plants/ha) et les Lauraceae (20 plants/ha) sont les plus denses.

\section{Structure diamétrique}

Pour les caféiers, elle prend l'allure d'une cloche avec un mode en classe 8-9 en basse et moyenne altitudes, et en classe 7-8 dans les autres zones. Ainsi, un regroupement de tiges s'observe dans les classes de diamètre comprises entre 7-9 cm (Figure 2).

La structure diamétrique des autres espèces ligneuses de basse altitude présente une forme exponentielle décroissante, avec un mode en classe < 10 constitué en majorité de légumineuses arborées; près de 76,3\% de ces espèces ligneuses sont concentrées dans les 
classes de diamètre $<20 \mathrm{~cm}$ (Figure 3). Dans les autres niveaux d'altitude, elle forme une structure en cloche. Les classes de diamètre comprises entre $20-40 \mathrm{~cm}$ sont les plus représentées en altitude intermédiaire $(42,3-48,3 \%$ des espèces ligneuses). En haute altitude, ce sont celles comprises entre $30-50 \mathrm{~cm}(58,5 \%$ des espèces ligneuses).

\section{Surface terrière}

Les agroforêts à base de caféiers de moyenne $\left(16,1 \mathrm{~m}^{2} / \mathrm{ha}\right)$ et haute altitudes $(11,9$ $\mathrm{m}^{2} / \mathrm{ha}$ ) possèdent les surfaces terrières les plus élevées. Hormis Canarium schweinfurthii (3,2 $\mathrm{m}^{2} / \mathrm{ha}$ ) en altitude moyenne inférieure, Cola spp. possède la surface terrière la plus élevée dans les autres niveaux. En altitude supérieure, les Sterculiaceae présentent les surfaces terrières les plus élevées $\left(4,8\right.$ et $\left.3,4 \mathrm{~m}^{2} / \mathrm{ha}\right)$; alors qu'en altitudes moyenne inférieure et basse, ce sont les Burseraceae (respectivement 4,3 et $2,0 \mathrm{~m}^{2} / \mathrm{ha}$ ).

\section{Composition floristique}

Occurrence parcellaire, dispersion et concentration

La Table 1 présente la fréquence des espèces complantées par classe d'occurrences dans les caféières arabica. En basse altitude, près de $70 \%$ des espèces présentent une occurrence parcellaire $<5$; le taux est de $57,4 \%$ en haute altitude contre $67,5 \%$ dans la zone intermédiaire.

Cola spp. (52\%), Dacryodes edulis (58\%), Persea americana (71\%) et Raphia mabilensis $(38 \%)$ présentent une forte occurrence parcellaire ; de plus, $14 \%$ d'agroforêts à base de caféiers arabica renferment au moins une tige ou une touffe de chacune de ces espèces. Par ailleurs, au moins une tige de Cola spp. et $P$. americana sont représentées dans $41 \%$ des agroforêts, dont $17 \%$ en moyenne altitude.

D. edulis est l'espèce la plus dispersée en basse altitude (16 apparitions sur 15 occurrences) tandis que $P$. americana représente les autres zones. Dracaena deisteliana (8 apparitions sur 1 occurrence) et Leucaena leucocephala (93 apparitions sur 11 occurrences) sont les plus concentrées respectivement en altitudes moyenne inférieure et basse. Si Ficus thonningii et Inga edulis le sont en haute altitude (4 apparitions sur 2 occurrences), en moyenne altitude, apparaît $F$. thonningii (6 apparitions sur 4 occurrences).

\section{Importance relative des espèces}

C. schweinfurthii et Cola spp. dominent en altitudes moyenne inférieure et basse respectivement avec $53,1 \%$ et $35,7 \%$ de la dominance de chacun des niveaux. Alors que, Croton macrostachyus en haute et $P$. americana en moyenne et haute altitudes (auxquelles sont associées Cola spp.) le sont, avec respectivement $67,9 \%$ et $65,1 \%$ de la dominance de chacun des niveaux (Table 2).

D. edulis et Cola spp. sont écologiquement les plus importantes respectivement en basse $(83,4)$ et moyenne altitudes $(127,7)$ et, tandis que $P$. americana (114,5 et 106) représente les autres niveaux.

\section{Importance relative des familles}

Les Burseraceae et les Sterculiaceae constituent les familles les plus dominantes : 50,6\%, 64,1\%, $53,6 \%$ et $49,3 \%$, respectivement dans les niveaux de basse, moyenne inférieure, moyenne et haute altitudes. Les trois familles écologiquement les plus importantes en basse altitude sont successivement les Mimosaceae (91\%), les Burseraceae $(51,3 \%)$ et les Sterculiaceae $(31,4 \%)$. En zone élevée, l'importance écologique des Burseraceae décroît, alors que celle des Sterculiaceae croît (Table 3). 

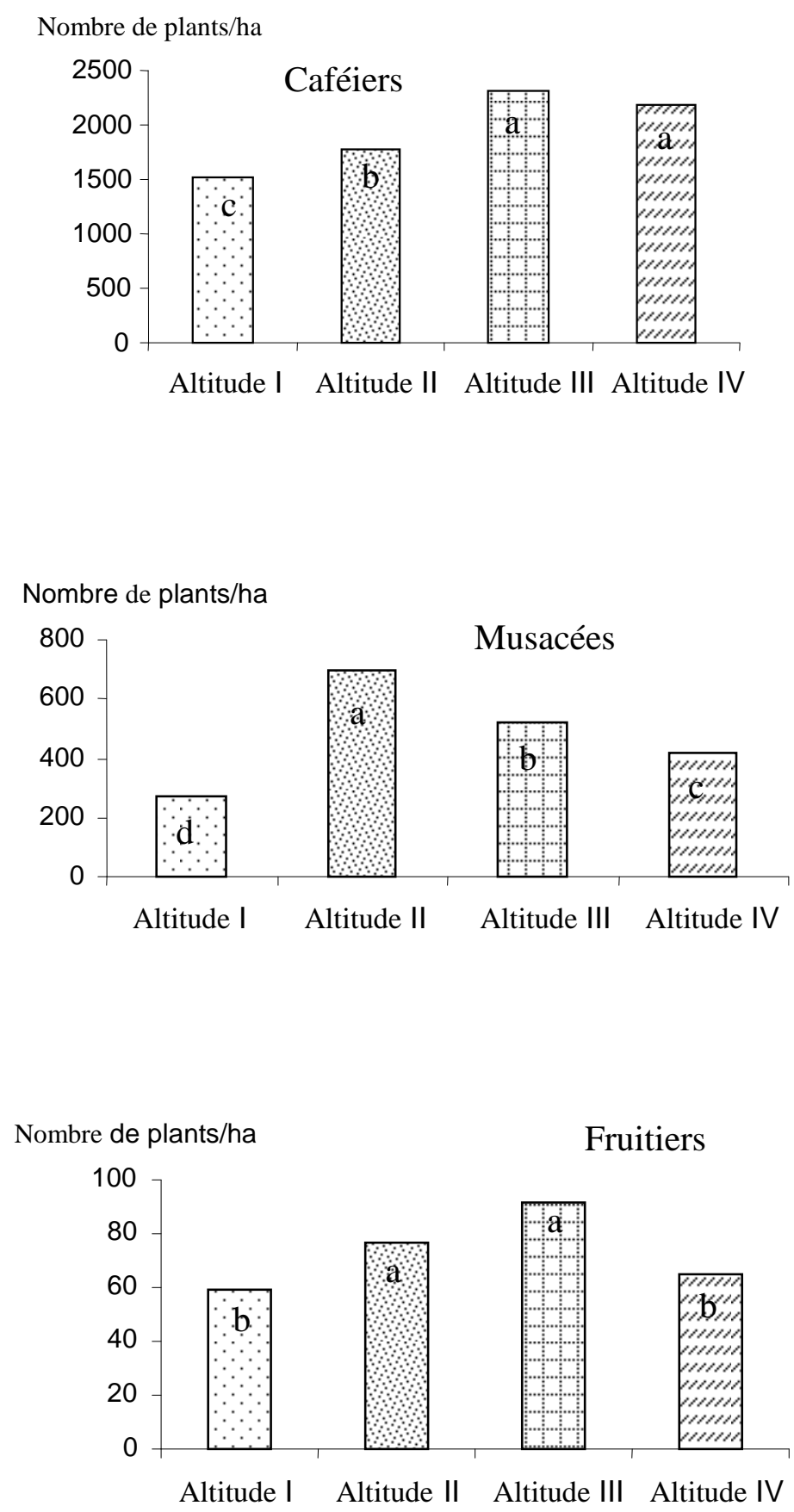
Nombre de plants/ha
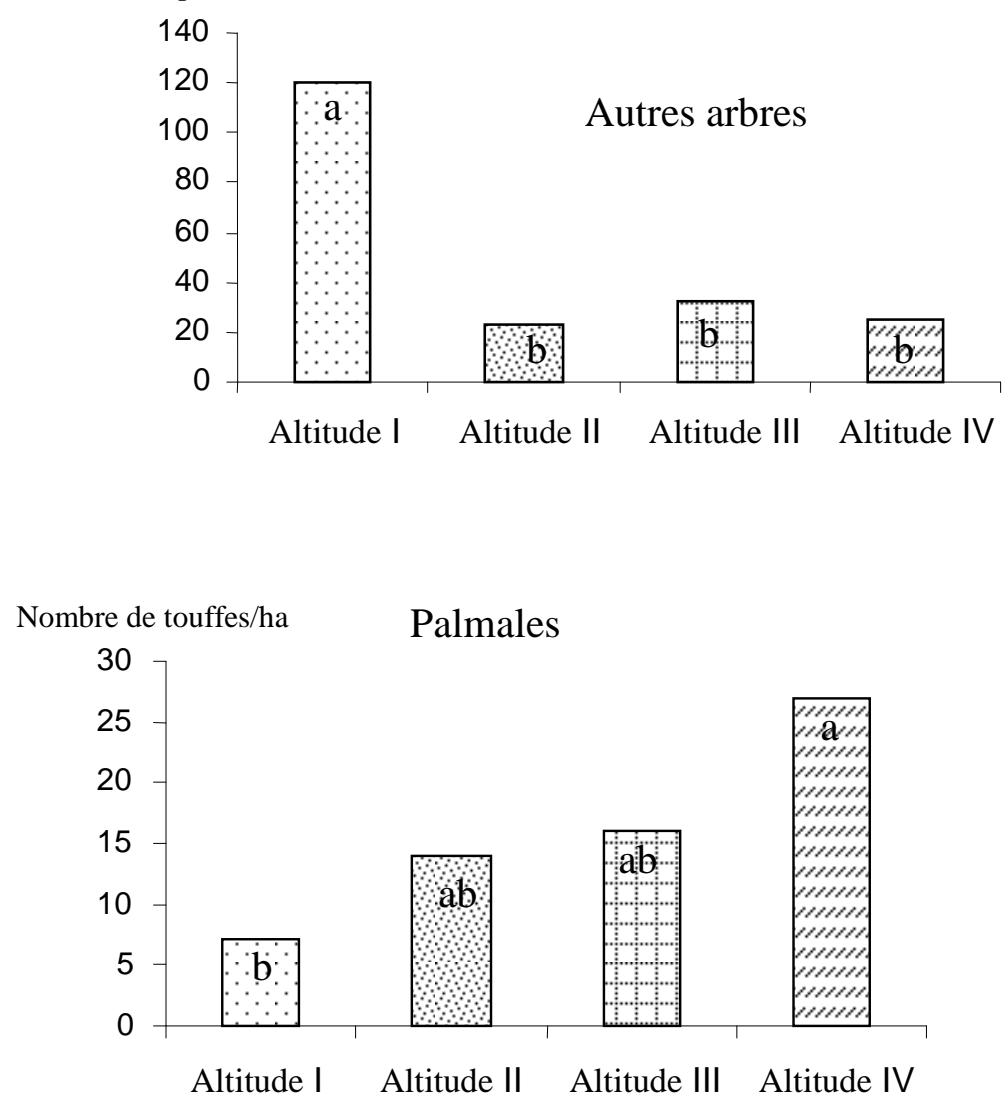

Figure 1 : Variation de la densité moyenne des groupes végétaux associés aux caféiers arabica suivant l'altitude (tiges/ha). Les histogrammes ne portant pas les mêmes lettres dans chacun des graphiques sont significativement différents à $\alpha=5 \%$.

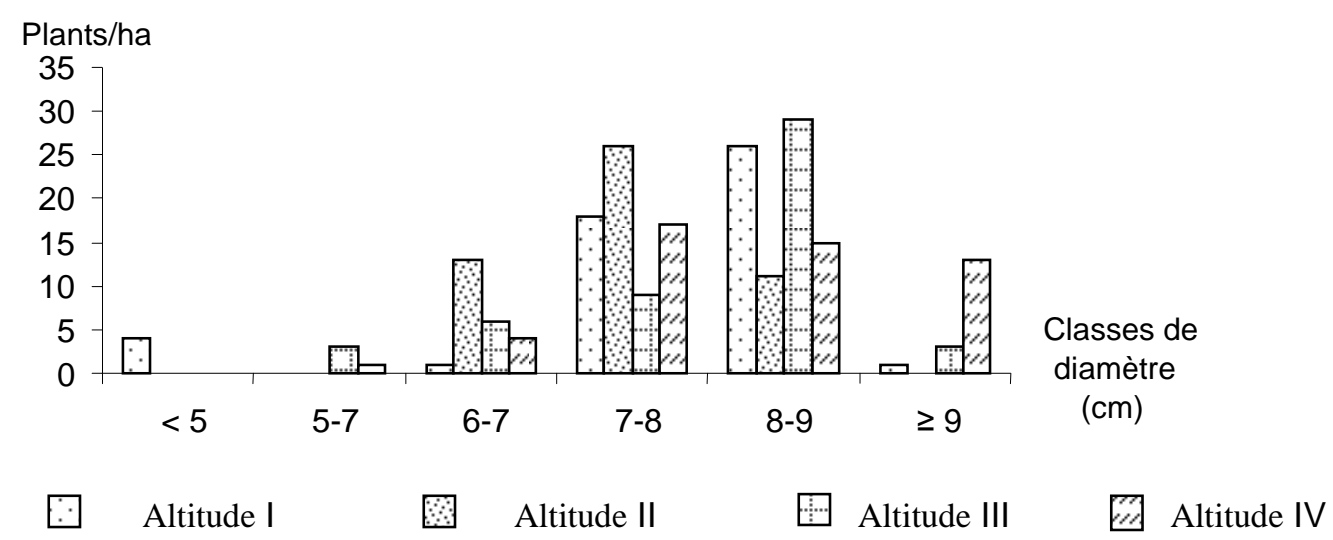

Figure 2 : Structure diamétrique des caféiers arabica par altitude. 


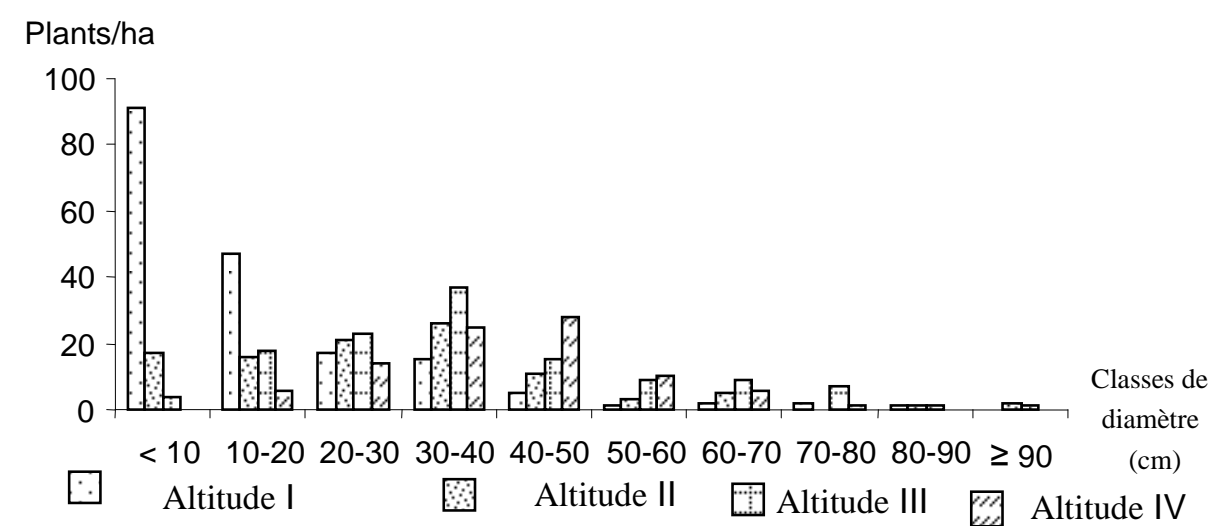

Figure 3 : Structure diamétrique des espèces ligneuses associées aux caféiers arabica.

\section{DISCUSSION \\ Densité moyenne \\ Caféiers arabica}

Dans les HPO, les densités des caféiers arabica sont significativement influencées par l'altitude. Celles trouvées en altitudes moyenne inférieure (1771 plants/ha) et basse (1521 plants/ha) sont plus réduites comparativement aux enquêtes de base du MINAGRI (Anonyme, 1981), et sensiblement inférieures à celles recommandées (2222 plants/ha) au Cameroun par Bouharmont (1981). En zones d'altitude supérieure, elles sont légèrement en dessous de celles recommandées par cet auteur (2500-2600 plants/ha). Très pluvieuses avec des vents violents, elles constituent des milieux de prédilection de l'anthracnose des baies (Mouen et al., 2008, 2010) et du ralentissement de la croissance des végétaux, d'où la forte densité prescrite.

Toutefois, ces normes prescrites sont remises en question face aux diverses contraintes et exigences actuelles des caféiculteurs en quête du bien - être.

\begin{abstract}
Musacées, fruitiers, Palmales et autres arbres
Les Musacées sont significativement plus denses en altitude moyenne inférieure (695 plants/ha). Comme le témoignent Nkendah et al. (2003), leur association dans les caféières est très largement prononcée dans cette région, comparativement aux études de Dallière et Dounias (1999), et, de Sonwa et al. (2000), respectivement sur les agroforêts de caféiers robusta dans la plaine Tikar et sur les cacaoyers au Sud-Cameroun.

Dans les agroforêts de caféiers à Kimisso Cameroun (Dallière et Dounias, 1999) et au Mexique (Soto-Pinto et al., 2001), les arbres d'ombrage y sont largement plus denses (respectivement 320 et 371,4 plants/ha) par rapport aux HPO du Cameroun.
\end{abstract}


M. A. MANGA et al. / Int. J. Biol. Chem. Sci. 7(4): 1474-1489, 2013

Table 1 : Fréquence absolue et occurrence parcellaire des espèces végétales dans les agroforêts de caféiers arabica par altitude.

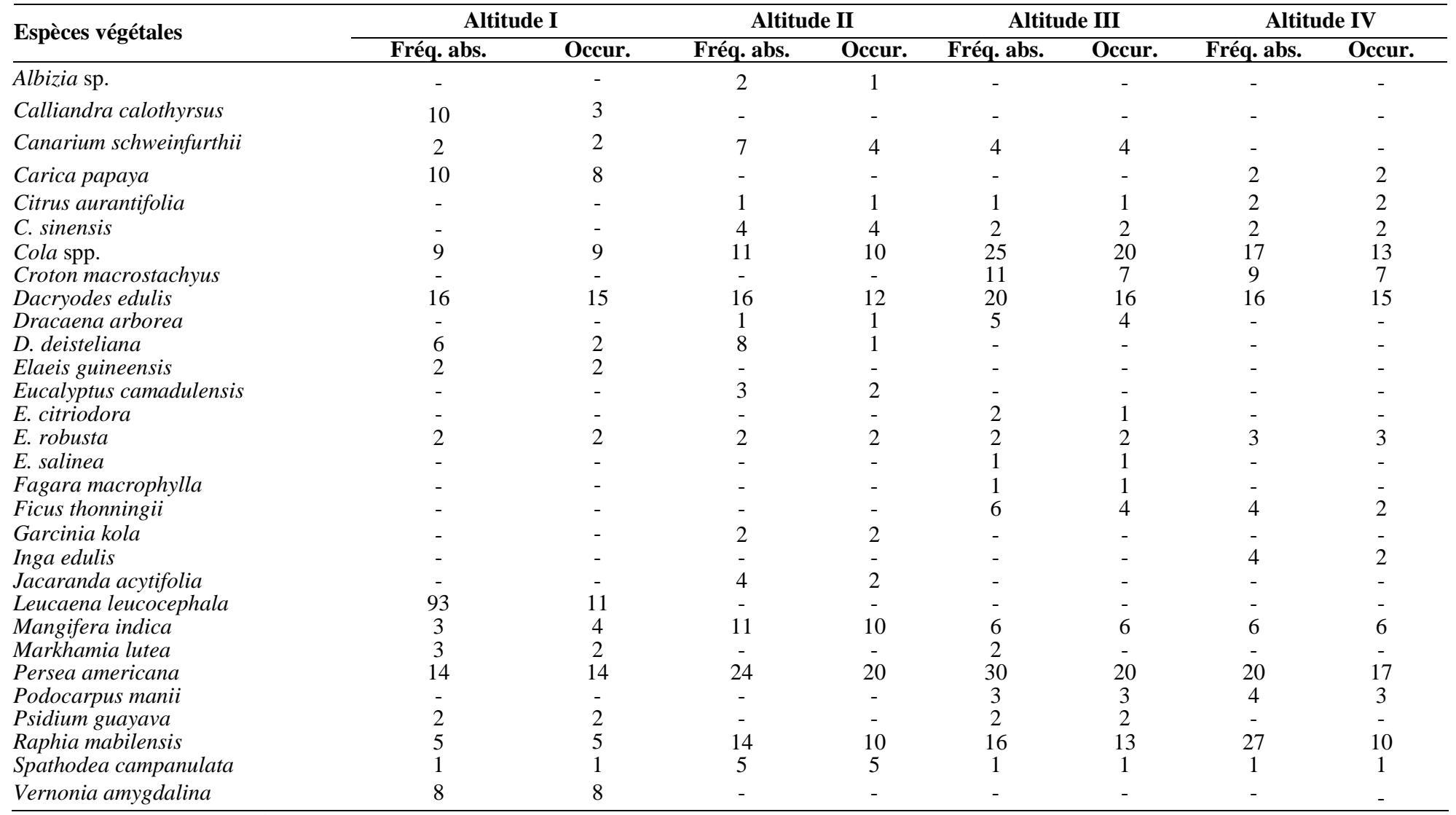

Fréq. abs. : fréquence absolue, Occur. : nombre d'occurrences, - : espèce absente. 
M. A. MANGA et al. / Int. J. Biol. Chem. Sci. 7(4): 1474-1489, 2013

Table 2 : Abondance, dominance et importance relative des espèces végétales associées aux caféiers arabica par altitude.

\begin{tabular}{|c|c|c|c|c|c|c|c|c|c|c|c|c|}
\hline \multirow{2}{*}{ Espèces végétales } & \multicolumn{4}{|c|}{ Abondance (\%) } & \multicolumn{4}{|c|}{ Dominance (\%) } & \multicolumn{4}{|c|}{ IVI (\%) } \\
\hline & Alt. I & Alt. & Alt. III & Alt. IV & Alt. I & Alt. & Alt. III & Alt. IV & Alt. I & Alt. II & Alt. III & Alt. IV \\
\hline Persea americana & 7,5 & 20,9 & 21,4 & 17,1 & 12,3 & 13,6 & 14,3 & 20,9 & 75,8 & 114,5 & 115,7 & 106,0 \\
\hline Cola spp. & - & 9,6 & 17,9 & 14,5 & 20,7 & 20,4 & 29,8 & 28,4 & 61,5 & 69,9 & 127,7 & 95,0 \\
\hline Dacryodes edulis & 8,6 & 13,9 & 14,3 & 13,7 & 14,8 & 11,0 & 9,9 & 14,9 & 83,4 & 72,9 & 88,2 & 88,5 \\
\hline Raphia mabilensis & - & 12,2 & 11,4 & 23,1 & - & - & - & - & - & - & 63,4 & 63,0 \\
\hline Croton macrostachyus & - & - & 7,9 & 7,7 & - & - & 23,8 & 15,8 & - & - & 59,7 & 51,5 \\
\hline Canarium schweinfurthii & - & - & - & - & 15 & 32,7 & 11,3 & - & - & 54,7 & - & - \\
\hline Leucaena leucocephala & 50 & - & - & - & 16 & - & - & - & 109,9 & - & - & - \\
\hline Mangifera indica & - & 9,6 & - & - & - & 6,5 & - & - & - & 56,0 & - & - \\
\hline Carica papaya & - & - & - & - & - & - & - & - & 38,9 & - & - & - \\
\hline Calliandra calothyrsus & 5,4 & & & & & & & & & & & \\
\hline Inga edulis & - & - & - & - & - & - & - & 4,7 & - & - & - & - \\
\hline
\end{tabular}

IVI : indice d'importance écologique pour les espèces végétales, Alt. : altitude, - non représentative, * : non estimée. 
M. A. MANGA et al. / Int. J. Biol. Chem. Sci. 7(4): 1474-1489, 2013

Table 3 : Abondance, dominance et importance relative des familles botaniques associées aux caféiers arabica par altitude.

\begin{tabular}{|c|c|c|c|c|c|c|c|c|c|c|c|c|}
\hline \multirow{2}{*}{ Familles botaniques } & \multicolumn{4}{|c|}{ Abondance (\%) } & \multicolumn{4}{|c|}{ Dominance (\%) } & \multicolumn{4}{|c|}{ FIV $(\%)$} \\
\hline & Alt. I & Alt. II & Alt. III & Alt. IV & Alt. I & Alt. II & Alt. III & Alt. IV & Alt. I & Alt. II & Alt. III & Alt. IV \\
\hline Sterculiaceae & 4,8 & 9,6 & 17,9 & 14,5 & 20,7 & 20,4 & 29,8 & 28,4 & 31,4 & 36,2 & 52,9 & 50,1 \\
\hline Burseraceae & 9,7 & 20,0 & 17,1 & 13,7 & 29,9 & 43,7 & 21,2 & 14,9 & 51,3 & 76,2 & 48,8 & 35,7 \\
\hline Lauraceae & 7,5 & 20,9 & 21,4 & 17,1 & 12,3 & 13,6 & 14,3 & 20,9 & 25,7 & 40,8 & 40,9 & 45,1 \\
\hline Arecaceae & - & 12,2 & 11,4 & 23,1 & 10,9 & - & - & - & 26,4 & - & - & 30,2 \\
\hline Euphorbiaceae & - & - & 7,9 & 7,7 & - & - & 23,8 & 15,8 & - & - & 36,9 & 30,6 \\
\hline Mimosaceae & 55,4 & - & - & - & 18,0 & - & - & 4,7 & 91,0 & - & - & - \\
\hline Myrtaceae & - & - & - & - & - & - & 2,9 & - & - & 22,3 & 28,9 & - \\
\hline Bignoniaceae & - & - & - & - & - & 5,5 & - & - & - & 25,8 & - & - \\
\hline Anacardiaceae & - & 9,6 & - & - & - & 6,5 & - & - & - & - & - & - \\
\hline
\end{tabular}


Contrairement aux études antérieures du MINAGRI dans les HPO (Anonyme, 1981), la prédominance des fruitiers s'oriente actuellement en altitude intermédiaire (Bamboutos, Bandjoun, Bansoa). Leur densité se rapproche de celle des caféières robusta de la plaine Tikar (80,1-95,5 plants/ha). Par contre en altitude extrême, elle est plus faible (59-65 plants/ha) et inférieure à celle des études d'Aulong et al. (2000) sur les cacaoyères enrichies homogènement (189 plants/ha) ou pures (260 plants/ha) du Sud-Cameroun, et de Sonwa (2004) sur les agroforêts de cacaoyers du Sud-Cameroun.

Les densités de Cola spp. (16,5 plants/ha) et Dacryodes edulis (10,6 plants/ha) dans les caféières robusta de Ngum (Dallière et Dounias, 1999), et de D. edulis (1,4-3,8 plants/ha) dans les cacaoyères du SudCameroun (Sonwa et al., 2000) sont nettement en dessous de celles des caféières arabica d'altitude supérieure. Par ailleurs, celle de Persea americana (22 plants/ha) dans les cacaoyères du Sud-Cameroun se rapproche de celles observées dans les caféières des HPO.

Les caféières de basse altitude sont les plus denses grâce à la forte présence de Leucaena leucocephala (93 plants/ha) et Calliandra calothyrsus (10 plants/ha) dans le Noun. L'implantation de ces légumineuses arborées est une pratique qui s'est développée afin d'enrichir continuellement le sol en matière organique, de le conserver et de transposer un microclimat proche de celui des systèmes naturels des caféiers arabica. Mais, leur densité est actuellement inférieure à celle recommandée (278 plants/ha) par Bouharmont (1981). Ainsi, la forte régression observée (75-96\%) traduit une forte pression anthropique exercée sur ces ligneux jugés concurrentiels pour les nouvelles spéculations alternatives, plus rentables (Nkendah et al., 2003; Kaffo et al., 2007 ; Uwizeyimana, 2009 ; Guétat-Bernard, 2011) telles que Lycopersicon esculentum, Phaseolus spp., Zea mays...

Dans les autres zones altitudinales des HPO, Dracaena deisteliana et Spathodea campanulata en moyenne inférieure, Croton macrostachyus, D. arborea et Ficus thonningii en moyenne, et, C. macrostachyus, Inga edulis et Podocarpus manii en haute altitudes sont couramment les arbres forestiers les plus retrouvés. Comme le soulignent Fotsing (1999b), la substitution d'Eucalyptus spp. dans cette région (jugée trop concurrentielle) est progressivement engagée par la forte présence de Podocarpus manii.

La production de bois d'œuvre dans ce milieu constitue principalement un revenu supplémentaire palliant aux fluctuations incessantes des cours mondiaux de café. Au Costa Rica, Boulay (2004) précise que les légumineuses arborées sont progressivement remplacées par du bois d'œuvre (75-125 plants/ha), afin de pallier au coût élevé de l'élagage de ces ligneux et de produire du bois plus rentable.

Concernant les Palmales, Elaeis guineensis ne se trouve qu'en basse altitude, alors que Raphia mabilensis augmente en densité en altitude croissante ; chaque caféière en possède au moins une touffe. Les études de Kaffo et al. (2007) soulignent l'importance de $R$. mabilensis dans la vie socio-économique des ménages de certaines régions (Bafou, Bamboutos, Djitutsa, Santa...).

\section{Structure diamétrique et surface terrière}

Le regroupement de la majorité des caféiers arabica en classe diamétrique $7-9 \mathrm{~cm}$ traduit ainsi l'absence d'un rajeunissement, suite à l'abandon de certaines opérations d'entretien : le recépage des vieilles tiges, la fertilisation, la replantation...

La structure diamétrique des autres espèces ligneuses de basse altitude est constituée en majorité de légumineuses arborées ; plus la 
classe diamétrique augmente, plus les espèces se raréfient. Quant aux zones d'altitude supérieure, plus l'altitude augmente, plus la majorité des espèces se décale vers les classes de diamètre supérieur. Les espèces ligneuses de diamètre < $20 \mathrm{~cm}$ sont majoritaires $(76,3 \%)$ comme dans les caféières mexicaines de Chiapas (Soto-Pinto et al., 2001).

La surface terrière des arbres d'ombrage est plus élevée en moyenne $\left(16,1 \mathrm{~m}^{2} / \mathrm{ha}\right)$ et haute altitudes $\left(11,8 \mathrm{~m}^{2} / \mathrm{ha}\right)$, compte tenu de la forte présence de Cola spp., Croton macrostachyus et Persea americana; les deux premiers couvrent $44,2-53,6 \%$ de la surface terrière des autres espèces ligneuses recensées en altitude supérieure. Mais, ces surfaces sont en dessous de celles de Soto-Pinto et al. (2000) dans les caféières mexicaines $\left(171 \mathrm{~m}^{2} / \mathrm{ha}\right)$, et de Sonwa (2004) dans les cacaoyères du SudCameroun $\left(30 \mathrm{~m}^{2} / \mathrm{ha}\right)$.

En effet, les caféiculteurs des HPO accordent peu d'intérêt aux grands arbres, lesquels nuiraient au développement des cultures vivrières et pérennes subvenant à leurs besoins (Guétat-Bernard, 2011). La majorité de ces arbres est localisée en altitude supérieure ; ils sont soit isolés dans les caféières, soit éléments constitutifs des haies anti-érosives. Ils jouent ainsi un rôle important dans la conservation des sols, l'ombrage des caféiers, le marquage foncier, le bois de chauffe... Vaast et Harmand (2002) soulignent cette même importance pour les caféières sud-américaines.

\section{Composition floristique}

Cette étude a permis de recenser 25 genres appartenant à 16 familles dominées par les Arecaceae, les Burseraceae, les Lauraceae et les Sterculiaceae (hormis les Musaceae et les Rubiaceae); par contre dans les caféières mexicaines, Soto-Pinto et al. (2000; 2001) ont identifié 32 familles dominées par les
Arecaceae, les Asteraceae, les Fabaceae et les Tiliaceae.

\section{Occurrence parcellaire, dispersion et concentration}

$\mathrm{Au}$ moins 57,4\% des espèces ligneuses recensées ont une occurrence parcellaire assez faible $(<5)$, comme dans les caféières mexicaines. Cette tendance est plus observée en basse altitude où existent d'importantes disparités mésologiques. Cola spp., Dacryodes edulis, P. americana et Raphia mabilensis sont les seules espèces présentant une occurrence assez forte, comme dans les enquêtes de base du MINAGRI (Anonyme, 1981).

De plus, $14 \%$ d'agroforêts à base de caféiers arabica renferment au moins une tige ou une touffe de chacune de ces espèces, et $41 \%$ de caféières présentent au moins une tige de Cola spp. et $P$. americana. Tandis qu'au Mexique, Soto-Pinto et al. (2001) indiquent qu'Inga spp. sont présentes dans $38 \%$ des caféières.

D. edulis est la plus dispersée en basse altitude tandis que $P$. americana représente les autres zones. En termes de concentration, Dracaena deisteliana et Leucaena leucocephala représentent respectivement les niveaux d'altitudes moyenne inférieure et basse.

Dans cette région, toutes les caféières arabica bénéficient de l'ombrage omniprésent de Musa spp. et de fruitiers, comme dans les caféières sud-américaines (Moguel et Toledo, 1999; Vaast et Harmand, 2002; Hall, 2003). Cela témoigne ainsi l'importante valeur de ces espèces associées non seulement pour la satisfaction des besoins quotidiens des caféiculteurs (Nkendah et al., 2003 ; Kaffo et al., 2007 ; Uwizeyimana, 2009 ; Guétat-Bernard, 2011), mais aussi leur rôle dans la stabilité des caféières.

\section{Importance relative des espèces et des familles}

L'index d'importance des espèces végétales rend plus explicite la prédominance des 
fruitiers dans les caféières des HPO du Cameroun. En moyenne et haute altitudes, ce sont Cola spp., Persea americana, Dacryodes edulis et Raphia mabilensis; précédée de $P$. americana, D. edulis accrô̂t écologiquement son importance en altitude inférieure. Les Sterculiaceae sont écologiquement les plus importantes en altitude supérieure; mais, le niveau d'altitude inférieure est représentée par les Burseraceae. Si Canarium schweinfurthii et Cola spp. dominent dans les caféières d'altitude inférieure; en altitude supérieure, ce sont Croton macrostachyus et $P$. americana (auxquelles sont associées Cola spp.).

Mais du Mexique (Soto-Pinto et al., 2000) au Nicaragua (Vaast et Harmand, 2002), Inga spp. présente une valeur importante (fixatrice d'azote, plate forme d'alimentation avienne) dans les caféières; elle est parfois supplantée par Gliricidia sepium dans les zones de basse altitude d'Honduras et du Nicaragua. Par ailleurs, Chin (2004) montre que Hevea brasiliensis au Brésil et Melia volkansii en Afrique de l'Est sont les mieux associées aux caféiers arabica.

\section{Conclusion}

Les travaux menés relèvent l'existence des disparités quant à la conduite floristique des agroforêts à base de caféiers arabica à tous les niveaux d'altitude. En effet, l'insertion des nouvelles spéculations culturales (vivrières, Palmales, fruitiers) valorisant mieux la terre et le travail est très accentuée; réduisant considérablement les surfaces caféières, particulièrement dans les niveaux de moyenne et basse altitudes. Cette étude permet ainsi de souligner les nécessités de la recherche d'une adaptation des normes de densités, face aux contraintes et exigences des caféiculteurs à la recherche d'un environnement viable et stable, d'un rajeunissement des vergers avec des semences améliorées et résistantes aux maladies, et afin de promouvoir une diversification raisonnée des divers groupes végétaux. Des recherches supplémentaires sont à poursuivre quant à l'appréhension de la diversité floristique et des stratégies de gestion des agroforêts à base de caféiers arabica.

\section{REFERENCES}

Anonyme. 1981. Enquête de base sur la zone du projet hauts plateaux de l'Ouest (avril 1980mars 1981). Rapport d'étude du Projet HPO-MINAGRI, Yaoundé, Cameroun, pp. 52-147.

Anonyme. 2009. Stratégie de développement de la filière café au Cameroun 2010-2015. Douala, Cameroun, 58 p.

Anonyme. 2012. Statistique de Production Nationale de Café au Cameroun. ONCC: Douala, Cameroun.

Aulong S, Duray S, Temple L. 2000. Dynamique et structure des agroforêts à agrumes au Centre du Cameroun. Fruits, 55(2) : 103114.

Bouharmont P. 1981. Expérimentation sur les dispositifs et les densités de plantation du caféier arabica au Cameroun. Café, Cacao, Thé, 25(4) : 243-261.

Bouharmont P. 1992. Sélection de la variété java et son utilisation pour la régénération de la caféière arabica au Cameroun. Café, Cacao, Thé, 36 (4) : 247-262.

Boulay A. 2004. Production de bois d'œuvre issu des systèmes agroforestiers caféiers au Costa Rica. Mémoire de fin d'études, Engref, Montpellier, France, 129 p.

Chin O. 2004. Plus d'ombre pour un meilleur café. Spore, 116 : 6.

Dalliere C, Dounias E. 1999. Agroforêts caféières et cacaoyères des Tikar (Cameroun central): structures, dynamiques et alternatives de développement. In $L a$ Gestion Durable des Forêts Denses 
Humides Africaines Aujourd'hui, Nasi R (ed). Cirad Forêt-Cifor-Iufro; 26 p.

Fotsing JM. 1999a. Croissance démographique et mise en culture des réserves forestières dans l'Ouest Cameroun. In Population and Deforestation in the Humid Tropics, Bilsborrow RE, Hogan D (eds). International Union for Scientific Study of Population: Liège, Belgique; 76-98.

Fotsing JM. 1999b. Excursion dans les agroforêts de l'Ouest: pays Bamiléké et Bamoun. Carnet de tournée. Colloque international l'homme et l'érosion du 9 au 19 décembre 1999, Yaoundé, 15p.

Gillison AN, Liswanti N, Budidarsono S, van Noordwijk M, Tomich TP. 2004. Impact of cropping methods on biodiversity in coffee agroecosystems in Sumatra, Indonesia. Ecology and Society, 9(2) : 7.

Guedje NM, Nkongmeneck BA, Lejoly J. 2002. Composition floristique et structure des formations à Garcinia lucida dans la région de Bipindi-Akom II (Sud-Cameroun). Acta Bot. Gallica, 149(2): 157-178.

Guétat-Bernard, H. 2011. Culture du café et transformations des rapports de genre en pays bamiléké au Cameroun. In $D u$ Grain à Moudre: Genre, Développement Rural et Alimentation, Verschuur C (ed). Actes des colloques genre et développement - Berne : DDC-Commission nationale suisse pour l'UNESCO; 167-188.

Hall S. 2003. Biodiversity conservation in agroecosystem: a comparaison of surfacedwelling beetle diversity in various shade coffee production system in Costa Rica. FES outstanding graduate student paper series 7 (2), Toronto University, Canada, $29 \mathrm{p}$.

Kaffo C, Kano MC, Temgoua YN. 2007. L'exploitation du raphia en économie de survie à l'ouest du Cameroun. Rapport des Recherches Africaines $\mathrm{N}^{\circ} 06$.

Latreille C, Poncy O, Ingrassia F, Crozier F. 2003. Analyse de la diversité floristique et de la structure du peuplement arboré dans l'optique de la caractérisation d'unités écologiques : la forêt du Mont tabulaire de la Trinité. Rapport de missions ONF, Paris, France; $154 \mathrm{p}$.

Moguel P, Toledo VM. 1999. Biodiversity conservation in traditional coffee systems of Mexico. Conserv. Biol., 13(1): 11-21.

Mouen BJA, Njiayouom I, Bieysse D, Ndoumbè NM, Cilas C, Nottéghem JL. 2008. Effect of shade on arabica coffee berry disease development: toward an agroforestry system to reduce disease impact. Ecology and Epidemiology, 98(12): 1320-1325.

Mouen BJA, Bieysse D, Nyassé S, Nottéghem JL, Cilas C. 2010. Role of rainfall in the development of coffee berry disease in Coffea arabica caused by Colletotrichum kahawae in Cameroon. Plant Pathology, 59: 324-329.

Nkendah R, Temple L. 2003. Pression démographique et efficacité technique des producteurs de banane plantain de l'ouest Cameroun. Cahiers Agricultures, 12(5): 333-339.

Sonwa DJ, Weise SF, Tchatat M, Nkongmeneck AB, Adesina A, Ndoye O, Gockowski J. 2000. Les agroforêts cacao : espace intégrant développement de la cacaoculture, gestion et conservation des ressources forestières au Sud-Cameroun. Papier présenté au $2^{\text {nd }}$ Symposium panafricain sur la gestion durable des ressources naturelles en Afrique, Ouagadougou, Burkina Faso.

Sonwa DJ. 2004. Biomass management and diversification within cocoa agroforest in the humid forest zone of southern 
Cameroon. Thèse de doctorat, Bonn University, Germany, $112 \mathrm{p}$.

Soto-Pinto L, Perfecto I, Castillo J, Hanson P, Caballero-Nieto JV. 2000. Shade effect on coffee production at the Northern tzetal zone of the State of Chiapas, Mexico. Agric. Ecosystem. Environ., 80 : 61-69.

Soto-Pinto L, Alvarado VR, Caballero-Nieto JV, Warnholtz GS. 2001. Woody plant diversity and structure of shade grown coffee in Northern Chiapas, Mexico. Rev. Biol. Trop., 49(3-4): 977-987.
Uwizeyimana L. 2009. Après le café, le maraîchage? Mutations des pratiques agricoles dans les Hautes Terres de l'Ouest Cameroun. Les Cahiers d'Outre-Mer, 247 : 331-344.

Vaast P, Harmand JM. 2002. Importance des systèmes agroforestiers dans la production de café en Amérique centrale et au Mexique. Rapport des Plantations, recherche, développement, 34-43. 